\title{
Risk factors for habitual snoring in children aged 2-14 years
}

\author{
Lei Lei $^{1}$, Zijing Jiang ${ }^{2}$, Jian Zou ${ }^{1}$, Yu Zhao ${ }^{1}$, Lingyu $\mathrm{Yu}^{1}$, and Ping Zhu ${ }^{1}$ \\ ${ }^{1}$ Sichuan University West China Hospital \\ ${ }^{2}$ Sichuan University West China Medical Center
}

February 25, 2021

\begin{abstract}
Abstract Background: Habitual snoring (HS), a prominent symptom of sleep-disordered breathing, is important to also consider the associated, multidimensional risk factors for HS in children. Aim: To identify risk factors for HS in children. Methods: A cross-sectional survey was performed in Chengdu. Children aged 2-14 years from four districts were randomly chosen to participate.Questionnaires were voluntarily completed by the children's guardians. Results: The survey included 926 boys and 622 girls, who were an average of 7.115 .25 years old. The sample included 463 habitual snorers (30.38\%), 683 occasional snorers (44.82\%), and 402 non-snorers (26.38\%). HS was found in $51.84 \%$ of preschool children and $26.6 \%$ of school children.Among the HS group, $31.3 \%$ had a maternal education of a college degree or higher and $86.6 \%$ had an immediate family member who snores. Breastfeeding duration among the HS group was significantly less than among the occasional snoring and non-snoring groups. History of symptoms of allergic rhinitis, rhinosinusitis, tonsillitis, and pneumonia/bronchitis in the past six months were associated with HS. Likewise, maternal smoking during pregnancy, maternal exposure to secondhand smoke during pregnancy , and child exposure to secondhand smoke were also associated with HS. Conclusion: The prevalence of HS was higher in preschool children. Having a mother with more education, a family history of snoring, a shorter period of breastfeeding, upper respiratory tract inflammation, and passive smoking are important risk factors for HS.
\end{abstract}

Title page

Risk factors for habitual snoring in children aged 2-14 years Lei Lei $^{1}$ Jian Zou $^{2}$ Zijing Jiang ${ }^{3}$ Yu Zhao ${ }^{4}$ Lingyu Yu ${ }^{1}$ Ping Zhu ${ }^{5}$

1.MD,PHD,Department of Otorhinolaryngology,Head\&Neck Surgery, West China Hospital, West China Medical School,Sichuan University,Sichuan,China;

2.MD,PHD,Associate Professor, Department of Otorhinolaryngology, Head\&Neck

3.Undergraduate,West China Medical School,Sichuan University,Sichuan,China;

4.MD,PHD,Professor,Department of Otorhinolaryngology,Head\&Neck Surgery,West China

Hospital,West China Medical School,Sichuan University,Sichuan,China;

5.MD,Department of Clinical Research Management,West China Hospital,Sichuan University

Corresponding Author :Jian Zou,MD,PHD,Associate Professor

Affiliation: Department of Otorhinolaryngology,Head\&Neck Surgery, West China Hospital, West China Medical School, Sichuan University, Chengdu,Sichuan, China

Postal/mail address: Department of Otorhinolaryngology, Head\&Neck Surgery, West China Hospital,West China Medical School, Sichuan University,37 Guoxuexiang, Wuhou District, Chengdu ,Sichuan,China 
Phone: +86 18980605883

Fax: +8602885422433

Email:65874911@qq.com

zip:610041

Keywords:Risk Factor;Habitual Snoring;Children

Running Head:Risk factors of Habitual Snoring

\title{
Risk factors for habitual snoring in children aged 2-14 years
}

\begin{abstract}
Background: Habitual snoring (HS), a prominent symptom of sleep-disordered breathing, is important to also consider the associated, multidimensional risk factors for HS in children.
\end{abstract}

Aim: To identify risk factors for HS in children.

Methods: A cross-sectional survey was performed in Chengdu. Children aged 2-14 years from four districts were randomly chosen to participate.Questionnaires were voluntarily completed by the children's guardians.

Results: The survey included 926 boys and 622 girls, who were an average of $7.11 \pm 5.25$ years old. The sample included 463 habitual snorers (30.38\%), 683 occasional snorers (44.82\%), and 402 non-snorers (26.38\%). HS was found in $51.84 \%$ of preschool children and $26.6 \%$ of school children.Among the HS group, $31.3 \%$ had a maternal education of a college degree or higher and $86.6 \%$ had an immediate family member who snores. Breastfeeding duration among the HS group was significantly less than among the occasional snoring and non-snoring groups. History of symptoms of allergic rhinitis, rhinosinusitis, tonsillitis, and pneumonia/bronchitis in the past six months were associated with HS. Likewise, maternal smoking during pregnancy, maternal exposure to secondhand smoke during pregnancy, and child exposure to secondhand smoke were also associated with HS.

Conclusion: The prevalence of HS was higher in preschool children. Having a mother with more education, a family history of snoring, a shorter period of breastfeeding, upper respiratory tract inflammation, and passive smoking are important risk factors for HS.

\section{KEYWORDS}

Risk Factor;Habitual Snoring;Children

\section{1 | INTRODUCTION}

Snoring is the most important and common manifestation of obstructive sleep apnea (OSA), which causes hypoxemia and hypercapnia from partial or complete obstruction of the upper airway during sleep ${ }^{1}$. Habitual snoring (HS), a prominent symptom of sleep-disordered breathing (SDB), is usually defined as the presence of loud snoring at least three nights per week ${ }^{2,3}$. Globally, HS is relatively common and has been reported in $2.4-45.6 \%$ of children ${ }^{2-4}$.

Recent research suggests that HS, even in the absence of OSA, is associated with cognitive, behavioral, and various psychosocial problems ${ }^{5,6}$. The clinical importance of HS as a potential marker of morbidity for OSA is increasingly recognized. Therefore, it is especially important to identify the multidimensional risk factors for 
HS among children, including age, obesity, exposure to respiratory infections, exposure to cigarette smoke, allergic rhinitis (AR), asthma, family history of snoring, prematurity, breastfeeding, living environment, and socioeconomic status. As such, using a large, population-based sample, we aimed to identify predisposing factors for HS, among the currently identified potential risk factors, in children of Chengdu.

\section{2 | MATERIALS AND METHODS}

\section{1 | Study sample}

A cross-sectional survey was performed in Chengdu, Sichuan Province in Southwest China. Four districts were chosen for participant recruitment, including a country village, a town, a county seat, and a city. Two kindergartens, two primary schools, and two junior middle schools from each district were randomly chosen to participate and the questionnaire was voluntarily completed by the children's guardians. The sample was children aged 2-14 years. Children with a history of neuromuscular disease, craniofacial syndromes, cerebral palsy, sickle cell disease, mucopolysaccharide storage disease, or immunodeficiency were excluded. Participants were also excluded if they had mental or physical impairment severe enough to cause abnormal behaviors, including congenital disease, intellectual disability, or a psychiatric disorder. The study was approved by the Biomedical Research Ethics Committee of the West China Hospital of Sichuan University. Written informed consent was obtained from each guardian before completing the questionnaire.

\section{2 | Questionnaire}

The investigators explained the purpose of the study and administered informed consent before administering the questionnaire. The following items from our parent-report questionnaire were used for analyses: (1) demographic factors including age, sex, race, weight, height, and district (city, suburb, county seat, town, or countryside village); (2) socioeconomic factors including family income and parental education; (3) delivery method (natural labor, Cesarean section), gestational age (premature birth, full-term birth, post-term birth), parental age at delivery, maternal pregnancy disease, and parental history of obstructive sleep apnea hypopnea syndrome; (4) symptoms of upper respiratory tract infection during the past six months, including tonsillitis, chronic rhinosinusitis, AR, and asthma; (5) passive smoke exposure (child exposed to secondhand smoke), maternal smoking and secondhand smoke exposure during the child's gestation; (6) and snoring; based on the OSA- 5 survey $^{7}$, participants were divided into three groups for comparisons (non-snorers $=$ "never", occasional snorers = "rarely" or "sometimes", and habitual snorers = "frequently" or "all the time."

\section{3 | Data analysis}

All analyses were performed using SPSS version 22.0. Data are presented as mean \pm SD, median (interquartile range), and percent, for parametric, nonparametric, and categorical data, respectively. Parametric and nonparametric variables were compared using one-way analysis of variance and Mann-Whitney tests. Categorical variables were compared using $\chi^{2}$ tests. Multivariate logistic regression analyses were subsequently performed to further confirm the association between potential risk factors and HS. The association was assessed in logistic regression models adjusted for age, sex, and BMI.

\section{3 | RESULTS}

\subsection{Participant characteristics and prevalence of habitual snorers}

Of the 1721 children enrolled, 197 questionnaires were excluded because: (1) the questionnaire was completed irregularly; (2) the child had a history of neuromuscular disease, craniofacial syndrome, cerebral palsy, sickle 
cell disease, mucopolysaccharide storage disease, or immunodeficiency; (3) the child had a mental or physical impairment severe enough to cause abnormal behaviors (including congenital disease, intellectual disability, or psychiatric disorder). The final 1524 questionnaires used for analyses represented a response rate of $88.56 \%$. The survey included 926 boys and 622 girls. The sample's average age was $7.11 \pm 5.25$ years, ranging from 2-14 years. Among these participants, 730, 282, 314, 119, and 103 lived in a city, a suburb, a county seat, a town, and a countryside village, respectively. Overall, there were 463 habitual snorers (30.38\%), 683 occasional snorers $(44.82 \%)$, and 402 non-snorers $(26.38 \%)$.

\section{2 | Risk factors for HS}

\subsection{1 | Social demography}

Social demographic risk factors were tabulated for each group and are shown in Table 1. HS morbidity was significantly higher in preschool children ( $<7$ years old) than in school children ([?]7 years old). Though boys $(31.53 \%, 292 / 926)$ had a slightly higher rate of HS than girls $(27.49 \%, 171 / 622)$, this difference was not statistically significant.

Fewer minority nationality children (2.6\%) than Han (97.4\%) participated, though there was no race difference in HS prevalence. Nor did HS morbidity differ based on living district (Table 1).

BMI scores were 16.62 (15.12-19.80), 16.63 (15.08-19.72), and 16.59 (15.15-19.96) for the HS group, occasional snoring group, and non-snoring group, respectively. Adjusting for age and gender, BMI did not differ among the snoring groups.

\subsection{2 | Socioeconomic factors}

According to the average household income statistics of Sichuan Province in 2019, we divided family monthly earning into six groups. No significant difference was found between the prevalence of HS and family monthly earning $(p=.21)$.

Paternal education did not differ between snoring groups $(p=.119)$. Higher maternal education was associated with greater HS prevalence $(p=.023)$; maternal college and undergraduate degrees were related to an increase in HS morbidity $(2.3 ; 95 \%$ CI, 1.1-4.7).

\subsubsection{Birth and heredity information}

Neither delivery method (natural labor vs. cesarean section) nor gestational age (premature, full-term, or post-term birth) was related to HS prevalence ( $p=.75$ and .11 , respectively). Nor were maternal or paternal ages at the child's birth related to HS prevalence $(p=.123$ and .373 , respectively).

In our sample, 77 mothers had pregnancy complications, including three highly prevalent diseases: diabetes, hypertension, and hypothyroidism. Interestingly, the prevalence of HS in children born to mothers with triple pregnancy complications was significantly lower compared with occasional and non-snoring (Table 2).

The average breastfeeding duration for the overall sample was $6.92+-5.51$ months. The between-groups difference in average breastfeeding duration was statistically significant $(p=.019)$. Breastfeeding duration $(6.07+-5.22$ months) in the HS group was significantly shorter than in the occasional snoring group (8.25 +- 5.91 months) and the non-snoring group (7.92 +- 6.12 months).

Parental loud snoring (considered a hereditary factor) was related to the HS prevalence $(p<.001)$ (1.16; $95 \%$ CI, 1.29-1.61).

\subsubsection{History of upper respiratory inflammation}

The prevalence of AR, nasosinusitis, and tonsillitis symptoms in the past six months for the HS, occasional, and non-snoring groups are listed in Table 3. AR symptoms (1.24; 95\% CI, 1.10-1.77), nasosinusitis 
symptoms (17.62; 95\% CI, 6.41-48.80), tonsillitis symptoms (19.66; 95\% CI, 9.51-55.38), and pneumonia/bronchitis symptoms (14.22; $95 \%$ CI, 5.42-38.01) within the past six months were related to HS incidence. However, only $20(1.3 \%)$ of the children had been diagnosed with asthma, which was unrelated to HS prevalence.

\subsection{5 | Passive smoking exposure}

In the HS group, the proportion of mothers with a history of smoking during pregnancy (43.1\%) was markedly higher than that in the non-snoring $(4.7 \%)$ and occasional snoring $(2 \%)$ groups. Both maternal exposure to secondhand smoke during pregnancy and child exposure were related to HS prevalence $(p<.00)$ (Table 4). To further investigate the relation between passive smoking and other factors and snoring, multivariate logistic regression analysis was undertaken, controlling for the potential confounders of age, gender, and upper respiratory inflammation in the fully adjusted model. Maternal smoking during pregnancy $(1.95 ; 95 \%$ CI, 1.63-2.34), maternal exposure to secondhand smoke during pregnancy (1.61;95\% CI, 1.08-2.39), and child exposure to secondhand smoke $(1.89 ; 95 \%$ CI, 1.56-2.23) were significant risk factors for HS.

\section{4 | DISCUSSION}

\section{1 | Social demography}

To our knowledge, this is one of the largest epidemiologic research studies carried out in Sichuan Province to examine the HS prevalence among children aged 2-14 years. We found a 30.38\% prevalence of HS in this age group. This HS prevalence is higher than the $10.5-27.39 \%$ previously reported in other Chinese studies ${ }^{2,8,9}$. A possible reason for this inconsistency might be heterogeneity between studies in participant age and area of residence. We speculate that another principal reason might be that our questionnaire was completed voluntarily. Guardians of children who snore habitually and have related symptoms may have been more likely to participate and complete the questionnaire.

Herein, there was an increased prevalence of HS in younger children. The prevalence rate in children under age 7 years was $51.84 \%$, and $26.6 \%$ in children 7 years and older. Two studies using large samples of children, by Li et al. ${ }^{2}$ and Anuntaseree et al. ${ }^{10}$, also showed interesting age differences in HS prevalence: a significant initial increase from ages 5-6 to 7 years and then a gradual decline. HS prevalence tended to decrease with age because of an increase in pharyngeal cross-sectional area with growth. This is consistent with the fact that lymphoid tissues in the larynx (i.e., adenoids, tonsils) gradually degenerate after age 7 years ${ }^{10,12}$.

Co-sleeping is also common among children under 7 years, which may have resulted in higher rates of reported snoring by their parents compared with the parents of older children.

We found a higher prevalence of HS in boys than in girls; however, this difference was not significant in our sample of 1524 children. In adults, HS is more common in men, for which the influence of sex hormones on respiratory control and/or body fat distribution has been suggested to play a crucial role. Clearly, these factors would be much less prominent in prepubertal children ${ }^{13}$. Papaioannou and colleagues have also reported that growing adenotonsillar tissue narrows the upper airway during early life, and adenotonsillar hypertrophy has been reported as a major determinant of OSA in children but not in adolescents ${ }^{14}$. Lumeng and Chervin have suggested that studies showing sex differences often include children over age 13 years, so there may be a potential mediating effect of puberty-related hormonal changes on the higher SDB prevalence in boys ${ }^{15}$.

Obesity has long been thought to be an important cause of snoring, but in our study, we did not find any significant difference between BMI among the three snoring groups or between the HS group and other groups. Two potential reasons for this result may be: (1) Obesity-related anatomic risk factors, other than BMI, including enlargement of parapharyngeal fat pads, lateral pharyngeal walls, the tongue, and total upper airway soft tissue ${ }^{16}$; (2) Some studies have shown that waist circumference (abdominal and hip fat), not 
BMI, are the most important causes of snoring in children ${ }^{17,18}$. Ours was an epidemiological survey study for which BMI was calculated based on parents' answers (height and weight values), and we were unable to measure abdominal and hip fat. Therefore, it is possible that BMI is not particularly reasonable for assessing risk for HS.

We found that the HS group had a higher proportion of children whose mother had a college-level or more education. In China, it is often the case that mothers are more focused on their children's growth and living habits compared with fathers. We speculate that mothers with a higher education had more access to information about HS-related diagnoses and symptoms and may have been more concerned about the impact of HS on their children's cognition and behavior.

In our survey, 77 mothers had pregnancy complications, including diabetes, hypertension, and hypothyroidism. An interesting finding was that the prevalence of HS was significantly lower in children born to mothers with three or more diseases during pregnancy. The mechanism of this cannot be explained at present.

Breastfeeding duration was significantly shorter within the HS group compared with the other groups. A meta-analysis by Sun et al. ${ }^{19}$ indicated that breastfeeding was associated with reduced risk of HS in children. There are two potential explanations underlying an impact of breastfeeding on HS risk. First, breastfeeding has a beneficial effect on mandibular development. Oral cavity features, such as high palates, retruded chin, and narrow dental arches are additional risk factors for snoring in children which may be partly prevented by breastfeeding. Second, breast milk may provide immunoglobulins that may help prevent viral respiratory infections and thus reduce the chronic upper airway inflammation and adenotonsillar hypertrophy which facilitate snoring ${ }^{20}$.

Family history of HS was another strong risk factor for childhood HS. Li et al. found that having one habitually snoring parent increased the child's HS risk by nearly 3.4 -fold ${ }^{13}$. Genetic factors contribute to craniofacial structure, body composition, and neuromuscular control of the upper airway and interact to produce a phenotype $\mathrm{e}^{21,22}$. A potential association has been suggested between the presence of a child's tonsillar hypertrophy and the presence of pharyngeal lymphoid tissue hypertrophy in their siblings and parents $^{21,23}$.

Numerous disorders have been associated with HS, including upper respiratory tract infection (asthma, AR, sinusitis, tonsillitis, and pneumonia/bronchitis) ${ }^{12,24}$. These may narrow the upper airway, causing inflammation that increases flow resistance and hence snoring propensity. Inflammatory mediators including histamine, CysLTs, IL 1 $\beta$, and IL-4, found in high levels in AR, also worsen sleep quality in those with OSA. Montelukast sodium and mometasone furoate have obvious anti-inflammatory effects, which has indirectly confirmed upper respiratory tract infection as an important cause of HS. Previous studies have reported a positive association between snoring and asthma. Herein, we could not detect a significant association between asthma and HS prevalence, likely due to the small number of positive cases.

In relation to the domestic environment, passive smoking was identified as a major risk factor for HS, consistent with other studies This was because we assert that maternal smoking during pregnancy, maternal exposure to secondhand smoke during pregnancy, and child exposure to secondhand smoke all result in child smoke exposure. Parent-reported smoking, particularly maternal smoking, has been associated with increased child snoring, and nighttime respiratory symptoms are exacerbated by such exposure ${ }^{12,25}$. Multivariate analyses by Subramanyam et al. show that children aged 3-18 years with severe OSA and tobacco smoke exposure have a 1.48 increased odds of developing OAHI than do those unexposed to tobacco smoke ${ }^{26}$. Studies by Urschitz et al. ${ }^{12}$ and $\mathrm{Zhu}^{27,28}$ have shown a positive association between household smoking and incidence of sleep-related hypoxia and HS, respectively. Cigarette smoke exposes the nasal and respiratory mucosa to large amounts of endotoxin, resulting in a potent inflammatory reaction ${ }^{29}$. It is likely that cessation of secondhand smoke exposure may also reduce oropharyngeal mucosal inflammation. Cigarette smoke causes ciliary dysfunction and proliferation of goblet cells with increased mucus production, in addition to mucosal inflammation ${ }^{30}$. Thus, inflammatory reactions and mucus production lead to respiratory tract 
narrowing and HS.

In conclusion, our epidemiological investigation shows that the prevalence of HS is higher in preschool children. Family history of snoring, having parents with less education, passive smoking, upper respiratory tract inflammation, and shorter breastfeeding durations are important HS risk factors.

\section{References}

1. Sateia MJ. International classification of sleep disorders, third edition: highlights and modifications. Chest . 2014;146:1387-1394.

2. Li S, Jin X, Yan C, Wu S, Jiang F, Shen X. Habitual snoring in school-aged children: environmental and biological predictors.Respir Res . 2010;11:114.

3. Sahin U, Ozturk O, Ozturk M, Songur N, Bircan A, Akkaya A. Habitual snoring in primary school children: prevalence and association with sleep-related disorders and school performance. Med Princ Pract . 2009,18:458-465.

4. Nafiu OO, Burke CC, Chimbira WT, Ackwerh R, Reynolds PI, Malviya S. Prevalence of habitual snoring in children and occurrence of peri-operative adverse events. Eur J Anaesthesiol . 2011;285:340-345.

5. Luo R, Galland BC, Gill AI, Dawes P, Schaughency E. Habitual snoring at age 3 years: links with parent-rated remembering in daily life and academic achievement at age 7 years. J Dev Behav Pediatr . 2018;39:144-153.

6. Li D, Liu D, Wang X, He D. Self-reported habitual snoring and risk of cardiovascular disease and all-cause mortality. Atherosclerosis . 2014;2351:189-195.

7. Soh HJ, Rowe K, Davey MJ, Horne RSC, Nixon GM. The OSA-5: development and validation of a brief questionnaire screening tool for obstructive sleep apnea in children. Int J Pediatr Otorhinolaryngol . 2018;113:62-66.

8. Li Y, Gao Q, Li L, Shen Y, Lu Q, Huang J, Sun C, Wang H, Qiao N, Wang C, Zhang H, Wang T. Additive interaction of snoring and body mass index on the prevalence of metabolic syndrome among Chinese coal mine employees: a cross-sectional study. BMC Endocr Disord . 2019;19:28.

9. Lu LR, Peat JK, Sullivan CE. Snoring in preschool children: prevalence and association with nocturnal cough and asthma. Chest . 2003;124:587-693.

10. Anuntaseree W, Sangsupawanich P, Mo-suwan L, Ruangnapa K, Pruphetkaew N. Prospective cohort study on change in weight status and occurrence of habitual snoring in children. Clin Otolaryngol . 2014;39:164-168.

11. Luo R, Schaughency E, Gill AI, Dawes PJ, Galland BC. Natural history of snoring and other sleepdisordered breathing (SDB) symptoms in 7-year-old New Zealand children: a follow-up from age 3. Sleep Breath . 2015;193:977-985.

12. Urschitz MS, Guenther A, Eitner S, Urschitz-Duprat PM, Schlaud M, Ipsiroglu OS, Poets CF. Risk factors and natural history of habitual snoring. Chest . 2004;126:790-800.

13. Li AM, Au CT, So HK, Lau J, Ng PC, Wing YK. Prevalence and risk factors of habitual snoring in primary school children. Chest . 2010;138:519-527.

14. Papaioannou G, Kambas I, Tsaoussoglou M, Panaghiotopoulou-Gartagani P, Chrousos G, Kaditis AG. Age-dependent changes in the size of adenotonsillar tissue in childhood: implications for sleep-disordered breathing. J Pediatr. 2013;162:269-274.e4. 
15. Lumeng JC, Chervin RD. Epidemiology of pediatric obstructive sleep apnea. Proc Am Thorac Soc . $2008 ; 5: 242-252$.

16. Larsson LG, Lindberg A, Franklin KA, Lundbäck B. Gender differences in symptoms related to sleep apnea in a general population and in relation to referral to sleep clinic. Chest . 2003;124:204-211.

17. Verhulst SL, Schrauwen N, Haentjens D, Suys B, Rooman RP, Van Gaal L, De Backer WA, Desager KN. Sleep-disordered breathing in overweight and obese children and adolescents: prevalence, characteristics and the role of fat distribution. Arch Dis Child . 2007;92:205-208.

18. de Sousa Caixêta JA, Saramago AM, de Cácia Pradella-Hallinan ML, Moreira GA, Tufik S, Fujita RR. Waist-to-height ratio distinguish obstructive sleep apnea from primary snoring in obese children.Sleep Breath . 2015;19:231-237.

19. Sun K, Guo Y, Zhang Y, Jiang X. Breastfeeding and risk of habitual snoring in children: A meta-analysis. Matern Child Nutr . 2019;15:e12799.

20. Bentley JP, Burgner DP, Shand AW, Bell JC, Miller JE, Nassar N. Gestation at birth, mode of birth, infant feeding and childhood hospitalization with infection. Acta Obstet Gynecol Scand . 2018;97:988-997.

21. Kalampouka E, Moudaki A, Malakasioti G, Panaghiotopoulou-Gartagani P, Chrousos G, Kaditis AG. Family history of adenotonsillectomy as a risk factor for tonsillar hypertrophy and snoring in childhood.Pediatr Pulmonol . 2014;49:366-371.

22. Wong ML, Sandham A, Ang PK, Wong DC, Tan WC, Huggare J. Craniofacial morphology, head posture, and nasal respiratory resistance in obstructive sleep apnoea: an inter-ethnic comparison. Eur J Orthod . 2005;27:91-97.

23. Friberg D, Sundquist J, Li X, Hemminki K, Sundquist K. Sibling risk of pediatric obstructive sleep apnea syndrome and adenotonsillar hypertrophy. Sleep . 2009;32:1077-1083.

24. Kukwa W, Guilleminault C, Tomaszewska M, Kukwa A, Krzeski A, Migacz E. Prevalence of upper respiratory tract infections in habitually snoring and mouth breathing children. Int $J$ Pediatr Otorhinolaryngol . 2018;107:37-41.

25. O'Callaghan F, O'Callaghan M, Scott JG, Najman J, Al Mamun A. Effect of maternal smoking in pregnancy and childhood on child and adolescent sleep outcomes to 21 years: a birth cohort study. BMC Pediatr . 2019;19:70.

26. Subramanyam R, Tapia IE, Zhang B, Mensinger JL, Garcia-Marcinkiewicz A, Jablonka DH, Galvez JA. Secondhand smoke exposure and risk of obstructive sleep apnea in children. Int $J$ Pediatr Otorhinolaryngol . 2020;130:109807.

27. Zhu Y, Au CT, Leung TF, Wing YK, Lam CW, Li AM. Effects of passive smoking on snoring in preschool children. J Pediatr . 2013;163:1158-1162.

28. Conway SG, Roizenblatt SS, Palombini L, Castro LD, Bittencourt LR, Silva RS, Tufik S. Effect of smoking habits on sleep. Braz J Med Biol Res . 2008;41:722-727.

29. Hsu WY, Chiu NY, Chang CC, Chang TG, Lane HY. The association between cigarette smoking and obstructive sleep apnea. Tob Induc Dis . 2019;17:27.

30. Weinstock TG, Rosen CL, Marcus CL, Garetz S, Mitchell RB, Amin R, Paruthi S, Katz E, Arens R. Predictors of obstructive sleep apnea severity in adenotonsillectomy candidates. Sleep . 2014;37:261-269.

\section{Hosted file}

TABLES.pdf available at https://authorea.com/users/398188/articles/510844-risk-factors-forhabitual-snoring-in-children-aged-2-14-years 